\section{Prevalência de sobrepeso e obesidade em adolescentes escolares do município de Fortaleza, Brasil}

\section{Prevalence of overweight and obesity among adolescent students in the city of Fortaleza, Brazil}

Lício de Albuquerque Campos 1

Álvaro Jorge Madeiro Leite 2

Paulo Cesar de Almeida 3

1,2 Departamento de Saúde Materno-Infantil. Universidade Federal do Ceará. Rua Prof. Costa Mendes, 1608. Fortaleza, CE, Brasil. CEP: 60.416-200. Fone: (85) 33668040 - Fax: (85) 33668046 E-mail: liciocampos@terra.com.br 3 Centro de Ciências da Saúde. Universidade Estadual do Ceará. Campus do Itaperi. Fortaleza, CE, Brasil.

\begin{abstract}
Objectives: to determine overweight and obesity prevalence in adolescent students in the Municipality of Fortaleza, Brazil and to estimate prevalence rates of public and private schools according to gender and age group (early and late adolescence).

Methods: a cross sectional study performed in the period of March to May 2003 comprised of 1158 adolescents, 571 from public schools and 587 from private schools. Overweight was defined when body mass index (BMI) was determined to be equal or above percentile 85 and under percentile 95; obesity was defined when BMI was determined as equal or over percentile 95, regardless of age and gender.

Results: total prevalence of overweight and obesity was of $19.5 \%$. In private schools, overweight/obesity reached $23.9 \%$, a rate higher than in public schools $(18.0 \%)(p=0.018)$. Overweight/ obesity prevalence in the male gender $(19.6 \%)$ was similar to the female gender $(19.0 \%) p=0.80)$, in early adolescence, prevalence was (24.1\%) higher than in late adolescence $(15.0 \%)(p<0.0001)$.

Conclusions: overweight and obesity prevalence in adolescent students of the city of Fortaleza was determined to be high, in private schools and higher in early adolescence with no difference between genders.
\end{abstract}

Key words Prevalence, Obesity, Adolescents, Students

\section{Resumo}

Objetivos: determinar a prevalência de sobrepeso e obesidade em adolescentes escolares do Município de Fortaleza, Brasil, e estimar a diferença entre a prevalência nas escolas públicas e privadas segundo sexo e faixa etária (adolescência precoce e tardia).

Métodos: estudo de corte transversal realizado no período de março a maio de 2003 com 1158 adoles centes, sendo 571 de escolas públicas e 587 de escolas privadas. Sobrepeso foi definido como indice de massa corporal (IMC) igual ou superior ao percentil 85 e inferior ao percentil 95; obesidade, IMC igual ou maior ao percentil 95, ambos para idade e sexo.

Resultados: a prevalência total de sobrepeso e obesidade foi 19,5\%. Nas escolas privadas, sobrepeso/obesidade alcançou 23,9\%, freqüência maior do que nas públicas $(18,0 \%)$ ( $p=0,018)$. A prevalência de sobrepeso/obesidade encontrada no sexo masculino $(19,6 \%)$ foi semelhante ao do feminino $(19,0 \%)$ ( $p=0,80)$; na adolescência precoce, a prevalência foi (24,1\%) maior do que a encontrada na adolescência tardia $(15,0 \%)(p<0,0001)$.

Conclusões: a prevalência de sobrepeso e obesidade em adolescentes escolares do município de Fortaleza mostrou-se elevada, sendo maior nas escolas privadas e na adolescência precoce, não ocorrendo diferença entre os sexos.

Palavras-chave Prevalência, Obesidade, Adolescentes, Estudantes 


\section{Introdução}

A obesidade vem aumentando sua prevalência de maneira expressiva em todo o mundo, fato que motivou a Organização Mundial da Saúde a qualificar tal situação como epidêmica. ${ }^{1}$ Esse aumento ocorreu em todas as faixas etárias, tanto nos países desenvolvidos quanto naqueles em desenvolvimento. ${ }^{2}$

Os diferentes critérios utilizados para a classificação de sobrepeso e obesidade em crianças e adolescentes nos estudos publicados dificultam uma avaliação mais precisa desta prevalência nos grupos de menor faixa etária, contudo, independentemente da classificação utilizada, a maioria dos estudos mostra aumento do sobrepeso e obesidade. ${ }^{3}$ De Onis e Blossner, 4 analisando dados mundiais sobre a avaliação do grau de desnutrição das populações, encontraram, em muitas sociedades em desenvolvimento, que o número de crianças obesas tornou-se gradativamente maior nas últimas décadas.

Todas as fases da infância são importantes para o estudo da obesidade, porém, a adolescência representa um período crítico para o desenvolvimento do excesso de peso. Nessa etapa do crescimento, o indivíduo adquire aproximadamente $25 \%$ da sua estatura final e $50 \%$ da sua massa corporal. 5 Deve ser considerado também que o risco de um adolescente obeso manter-se assim até a idade adulta chega a aproximadamente $80 \% .6$

No Brasil, várias investigações demonstram alterações significativas no perfil nutricional da população, em razão do processo denominado transição nutricional.7 Essas modificações são constatadas nos inquéritos domiciliares nacionais e regionais realizados no período de 1975-1997, apontando uma diminuição da desnutrição no país para alguns estratos sociais e regiões. ${ }^{8}$

Neutzlin, ${ }^{9}$ analisando os dados nutricionais coletados pelo Instituto Brasileiro de Geografia e Estatística (IBGE), na Pesquisa Nacional sobre Saúde e Nutrição (PNSN), em 1989, encontrou a prevalência de sobrepeso de 7,7 \% para a população de adolescentes. Considerando os dados da Pesquisa sobre Padrões de Vida, inquérito domiciliar realizado pelo IBGE, em 1998 entre 1996 e 1997, a prevalência de sobrepeso e obesidade entre adolescentes de 15 a 19 anos foi de 8,45\% para o Nordeste e de $11,53 \%$ para o Sudeste. 10

Em Fortaleza, capital do Estado do Ceará, no Nordeste do Brasil, foi realizado um estudo acerca de sobrepeso e obesidade em escolas públicas, evidenciando a prevalência de $11,4 \%$ nos adolescentes entre 10 e 14 anos. 11 No entanto, informações sobre o estado nutricional de toda a faixa etária de adolescentes, com amostra representativa das escolas públicas e privadas, não estão disponíveis.

Este estudo tem por objetivo determinar a prevalência de sobrepeso e obesidade em adolescentes escolares e estimar a diferença entre a prevalência nas escolas públicas e privadas segundo sexo e faixa etária (adolescência precoce e tardia).

\section{Métodos}

Estudo transversal, utilizando como amostra adolescentes na faixa de idade de 10 a 19 anos, matriculados nas escolas da rede de ensino público e privado do município de Fortaleza, Ceará, Brasil, realizado no período de março a maio de 2003 . As séries estudadas correspondem ao ensino fundamental da quinta a oitava séries e ensino médio.

Segundo dados do Instituto Nacional de Estudos e Pesquisas Educacionais, 12 foram matriculados 377.461 alunos entre a quinta série do ensino fundamental e terceiro ano do ensino médio, nas escolas públicas (federais, estaduais e municipais) e privadas do município de Fortaleza. Dessa população, aproximadamente $75 \%$ dos adolescentes estão nos colégios públicos e o restante nos privados. O cálculo do tamanho amostral foi realizado pelo programa EpiInfo versão 6.04. Utilizou-se a população de adolescentes já referida, com uma expectativa de freqüência de sobrepeso/obesidade de $11 \%$, tendo como base um estudo prévio realizado no município de Fortaleza, 11 com erro amostral de $2 \%$ e o nível de significância foi estabelecido em $5 \%$. Com base nesses dados, obteve-se uma população de estudo de 938, contudo, para prováveis perdas de informações, empregou-se uma margem de segurança, sendo o estudo realizado com 1158 adolescentes, 571 nas escolas públicas e 587 nas particulares.

O município de Fortaleza é composto por seis regionais com populações numericamente semelhantes. A amostra para cada grupo de escolas obedeceu a seguinte seqüência: nas escolas da rede pública, através de amostragem aleatória simples, selecionou-se uma escola em cada regional. Para representar as escolas particulares, foi feita inicialmente uma divisão por valor monetário das mensalidades, sendo estabelecidos quatro grupos: grupo $1=$ até $50 \%$ do salário mínimo; grupo $2=$ de $1 / 2$ até 1 salário mínimo; grupo $3=$ de 1 até 1 e $1 \frac{1}{2}$ salário mínimo e grupo 4 = acima de 1 e 1/2 salário mínimo. Após a determinação desses grupos, foi escolhida por sorteio aleatório uma escola em cada grupo. Através desses critérios, foram selecionadas seis 
escolas públicas e quatro particulares.

Os alunos foram escolhidos conforme uma tabela de números randômicos gerada no programa Epi-Info. A seleção dos alunos em cada sala de aula foi correspondente à ordenação na caderneta escolar. $\mathrm{Na}$ ausência de determinado estudante, foi selecionado o próximo aluno da tabela. Nas quatro escolas particulares, foi sorteada uma turma de cada série e aproximadamente 20 alunos de cada turma. Nas seis escolas públicas, foram escolhidos aleatoriamente sete turmas e aproximadamente 14 alunos foram sorteados em cada uma.

O projeto de pesquisa foi aprovado pelo Comitê de Ética em Pesquisa (COMEPE) do Complexo Hospitalar da Universidade Federal do Ceará.

As informações e medidas antropométricas foram obtidas nas próprias escolas, por médicos, nutricionistas, educadores físicos e alunos de Medicina devidamente treinados para este fim. As avaliações foram realizadas, após consentimento dado por escrito pelos alunos de 18 a 19 anos e, abaixo dessa faixa etária, pelos pais.

As medidas antropométricas foram realizadas com o emprego de balanças portáteis digitais, modelo Plenna, que utilizam a medida por pressão e tecnologia microeletrônica, com precisão de $0,1 \mathrm{~kg}$ calibradas até $150 \mathrm{~kg}$, e antropômetros portáteis do tipo person-check, da marca KaWe, com precisão de $1 \mathrm{~cm}$.

A avaliação nutricional foi realizada através do Índice de Massa Corpórea (IMC = peso em quilos dividido pela altura em metros ao quadrado). Considerou-se como ponto de corte percentil igual ou maior que 85 e menor que 95 para sobrepeso, e para obeso, o percentil igual ou maior que 95, tendo como referência as tabelas de Must et al.,13 com base nos valores antropométricos da população norteamericana, coletados na National Health and Nutrition Examination Survey I (NHANES). Adotou-se a expressão sobrepeso/obesidade para definir o excesso de massa corporal correspondendo aos critérios da World Health Organization (WHO), ${ }^{14}$ que estabelece o termo "risco de sobrepeso" para os adolescentes com IMC maior ou igual ao percentil 85 da população americana.

A classificação das faixas etárias utilizadas corresponde a duas fases, adolescência precoce e tardia. A primeira, compreendendo a faixa etária de 10 aos 14 anos, inclui o início das mudanças puberais e a segunda, de 15 aos 19 anos, que é a fase do término do crescimento e do desenvolvimento morfológico. 15

O processamento de dados e as análises estatísticas foram realizados através do programa Epi-Info versão 6.04 .

Foram calculadas as prevalências de sobrepeso e obesidade e seus respectivos intervalos de confiança de $95 \%$. Na determinação das prevalências que envolveram todos os adolescentes das escolas públicas e privadas, realizou-se a padronização pelo método direto, visto que $75 \%$ dos escolares do município de Fortaleza estão nos colégios públicos e $25 \%$ nos privados. Assim, eliminou-se um possível efeito de confundimento, pois com uma combinação ponderada, a população estudada apresentou o mesmo peso da população padrão. O teste do $\chi^{2}$, com nível de significância de $5 \%$, foi usado para avaliar as diferenças observadas nas taxas de prevalências entre os vários grupos e, também, as razões de prevalências e seus respectivos intervalos de confiança.

\section{Resultados}

Foram avaliados 1.158 adolescentes de 10 a 19 anos, sendo $555(47,9 \%)$ do sexo masculino e $603(52,1 \%)$ do sexo feminino.

A prevalência de sobrepeso/obesidade para toda a amostra foi de $19,5 \%$ (IC95\% = 17,3 a 21,9\%). Essa prevalência foi determinada pela padronização do método direto, uma vez que nas escolas públicas estudam $75 \%$ dos escolares do município de Fortaleza e $25 \%$ nos privados.

Observou-se maior freqüência de sobrepeso/ obesidade nas escolas privadas quando comparadas às públicas (Tabela 1).

A distribuição de prevalência de sobrepeso/obesidade por sexo não mostrou diferença significativa, sendo no masculino de $20 \%$ e no feminino de $19 \%$. Em relação à faixa etária, foi encontrada diferença estatisticamente significante entre a prevalência de sobrepeso/obesidade, sendo na adolescência precoce (10 - 14 anos) de $24,7 \%$ e na tardia (15 - 19 anos) de $15,1 \%$ (Tabela 1 ).

$\mathrm{Na}$ avaliação das escolas públicas, não se identificou diferença na prevalência de sobrepeso/obesidade em relação ao sexo e à faixa etária, já nas escolas privadas foi constatada diferença estatisticamente significante (Tabela 2).

As maiores taxas de sobrepeso/obesidade foram encontradas nos adolescentes de 10 a 14 anos, do sexo masculino, estudantes das escolas privadas (Tabela 2).

Comparando-se as frequiências de sobrepeso/obesidade entre os jovens dos colégios privados e públicos, foi evidenciado que, no sexo masculino, os adolescentes das escolas particulares têm maiores 
Tabela 1

Prevalência de sobrepeso/obesidade em adolescentes escolares de colégios públicos e privados. Fortaleza, Ceará, 2003.

\begin{tabular}{|c|c|c|c|c|c|c|}
\hline \multirow[t]{2}{*}{ Variáveis } & \multicolumn{6}{|c|}{ Sobrepeso/Obesidade } \\
\hline & $\mathrm{n}$ & $n$ & $\%$ & $p$ & $\mathrm{RP}$ & IC95\% \\
\hline \multicolumn{7}{|l|}{ Escolas } \\
\hline Públicas & 571 & 103 & 18,0 & 0,015 & 1,32 & $1,05-1,66$ \\
\hline Privadas & 587 & 140 & 23,9 & & & \\
\hline \multicolumn{7}{|l|}{ Sexo } \\
\hline Masculino & 555 & 135 & 20,0 & 0,56 & 0,56 & $0,89-1,24$ \\
\hline Feminino & 603 & 108 & 19,0 & & & \\
\hline \multicolumn{7}{|l|}{ Idade } \\
\hline 10-14 anos & 579 & 158 & 24,7 & $<0,0001$ & 1,64 & $1,39-1,94$ \\
\hline 15-19 anos & 579 & 85 & 15,1 & & & \\
\hline
\end{tabular}

Nas prevalências que avaliam o sexo e idade foi utilizada a padronização pelo método direto, visto que $75 \%$ dos escolares do município de Fortaleza estão nos colégios públicos e $25 \%$ nos privados.

Tabela 2

\begin{tabular}{|c|c|c|c|c|c|c|c|c|}
\hline \multirow[t]{3}{*}{ Variáveis } & \multicolumn{7}{|c|}{ Sobrepeso/Obesidade } & \\
\hline & \multicolumn{4}{|c|}{ Escolas Públicas } & \multicolumn{4}{|c|}{ Escolas Privadas } \\
\hline & $\mathrm{n}$ & $\mathrm{n}$ & $\%$ & $p$ & $\mathrm{n}$ & $\mathrm{n}$ & $\%$ & $p$ \\
\hline \multicolumn{9}{|l|}{ Sexo } \\
\hline Masculino & 259 & 40 & 15,4 & 0,17 & 296 & 95 & 32,1 & $<0,0001$ \\
\hline Feminino & 312 & 63 & 20,2 & & 291 & 45 & 15,5 & \\
\hline \multicolumn{9}{|l|}{ Idade } \\
\hline $10-14$ anos & 235 & 51 & 21,8 & 0,07 & 344 & 107 & 31,1 & $<0,0001$ \\
\hline $15-19$ anos & 336 & 52 & 15,5 & & 243 & 33 & 13,6 & \\
\hline \multicolumn{9}{|l|}{ Masculino } \\
\hline 10-14 anos & 91 & 17 & 18,7 & 0,28 & 169 & 72 & 42,6 & $<0,0001$ \\
\hline $15-19$ anos & 168 & 23 & 13,7 & & 127 & 23 & 18,1 & \\
\hline \multicolumn{9}{|l|}{ Feminino } \\
\hline 10-14 anos & 144 & 34 & 23,6 & 0,16 & 175 & 35 & 20,0 & 0,008 \\
\hline $15-19$ anos & 168 & 29 & 17,3 & & 116 & 10 & 8,6 & \\
\hline
\end{tabular}


Comparação das prevalências de sobrepeso/obesidade entre adolescentes escolares de colégios públicos e privados, segundo a distribuição por sexo e faixa etária. Fortaleza, Ceará, 2003.

\begin{tabular}{|c|c|c|c|c|c|c|}
\hline \multirow[t]{2}{*}{ Variáveis } & \multicolumn{6}{|c|}{ Sobrepeso/Obesidade } \\
\hline & $\mathrm{n}$ & $\mathrm{n}$ & $\%$ & $p$ & $\mathrm{RP}$ & IC $95 \%$ \\
\hline \multicolumn{7}{|l|}{ Sexo Masculino } \\
\hline Escola Pública & 259 & 40 & 15,4 & $<0,0001$ & 2,08 & $1,49-2,89$ \\
\hline Escola Privada & 296 & 95 & 32,1 & & & \\
\hline \multicolumn{7}{|l|}{ Sexo Feminino } \\
\hline Escola Pública & 312 & 63 & 20,2 & 0,16 & 1,31 & $0,92-1,85$ \\
\hline Escola Privada & 291 & 45 & 15,5 & & & \\
\hline \multicolumn{7}{|l|}{ 10-14 anos } \\
\hline Escola Pública & 235 & 51 & 21,8 & 0,016 & 1,43 & $1,07-1,91$ \\
\hline Escola Privada & 344 & 107 & 31,1 & & & \\
\hline \multicolumn{7}{|l|}{ 15-19 anos } \\
\hline Escola Pública & 336 & 52 & 15,5 & 0,60 & 1,14 & $0,76-1,91$ \\
\hline Escola Privada & 243 & 33 & 13,6 & & & \\
\hline
\end{tabular}

taxas de sobrepeso/obesidade que os das escolas públicas; no sexo feminino, não foi encontrada diferença estatisticamente significante (Tabela 3 ). Considerando a avaliação das faixas etárias, foi constatado que, na adolescência precoce $(10$ a 14 anos), os jovens das escolas privadas têm mais excesso de peso ao serem comparados aos dos colégios públicos; na adolescência tardia (15 a 19 anos), contudo, não foi detectada diferença estatisticamente significante da prevalência de sobrepeso/obesidade entre os dois tipos de escolas (Tabela 3).

Ao se analisar as prevalências de sobrepeso/obesidade entre os adolescentes, levando em consideração sexo, faixa etária e tipo de escola (públicas e privadas), encontraram-se importantes diferenças. Adolescentes masculinos de 10 a 14 anos das escolas particulares apresentaram prevalência de sobrepeso/obesidade de 42,6\% (IC95\% : 35,1 $50,4)$, enquanto que, nos de colégios públicos, a prevalência foi de 18,7\% (IC95\% : 11,6 - 28,5), ocorrendo diferença estatisticamente significante ( $p$ $=0,0001)$. Adolescentes femininas de 15 a 19 anos das escolas públicas apresentaram maior prevalência de sobrepeso/obesidade $(17,3 \%)$ que as das particulares $(8,6 \%) ;(p=0,037)$.

\section{Discussão}

A importância crescente do problema da obesidade nas crianças e adolescentes justifica maior vigilância sobre esse grupo etário, tornando-se necessárias iniciativas, ainda incipientes no país, objetivando o controle do sobrepeso e obesidade. 8

Neste estudo, a prevalência de sobrepeso e obesidade em adolescentes escolares foi elevada $(19,5 \%)$, comparando-se com o último inquérito nutricional realizado na região Nordeste em 1997 , em que, na faixa etária de 15 a 19 anos foi encontrada esta prevalência de 8,45\%.10 Considerando a elevada taxa de crianças nas escolas, o colégio tornou-se o local adequado para avaliações epidemiológicas, o que faz esta amostra representativa para toda a população de adolescentes de uma determinada base territorial.

$\mathrm{Na}$ adolescência, o excesso de peso pode resultar em alterações metabólicas importantes, dependendo da sua duração e gravidade, cujas conseqüências ocorrem mais nos adultos. $16 \mathrm{O}$ adolescente obeso tem maior risco para algumas doenças e distúrbios psicossociais de grande importância nessa fase de estruturação da personalidade. ${ }^{17}$

Os dados obtidos nas escolas estudadas revelam que a prevalência de sobrepeso/obesidade foi maior nos adolescentes matriculados nas escolas privadas. 
Assim, pode-se supor que a condição socioeconômica pode representar um fator contribuinte para tal diferença.

A prevalência de sobrepeso/obesidade não foi diferente entre os adolescentes masculinos e femininos, contudo, na avaliação das escolas públicas e privadas, separadamente, não se observou diferença nas escolas públicas, porém nas particulares as meninas apresentaram menor freqüência de sobrepeso/obesidade. Uma possível explicação para essa diferença é que a preocupação do excesso de peso é maior nas meninas com melhores condições socioeconômicas. 10

Comparando a adolescência precoce (10 - 14 anos) com a tardia (15 - 19 anos), identificou-se maior proporção de sobrepeso/obesidade para as mais jovens. Provavelmente em decorrência de muitos adolescentes nessa faixa etária ainda estarem passando pelas diversas fases do desenvolvimento puberal, e esse excesso de peso poderia ser compensado pelo crescimento. 18

A definição da obesidade infantil não encontra ainda unanimidade na comunidade científica, causando dificuldades na comparação entre os diversos estudos. Os valores de estudo do IMC escolhido para este trabalho foram os de Must et al.,13 por ser o recomendado pela $\mathrm{WHO}^{14}$ e também por ter sido amplamente utilizado em estudos nacionais, principalmente os que avaliam com amostra probabilística a população nacional.9,10

A utilização da população americana, como valor de referência, para determinar o sobrepeso, pode subestimar os dados em países em desenvolvimento como o Brasil.14 Segundo Sardinha et al.,19 embora a proposta da $\mathrm{WHO}^{14}$ utilize o corte no percentil 85 do IMC para ser usado no rastreamento de adolescentes com risco de obesidade, o ponto de corte de maior equilíbrio entre a sensibilidade e especificidade estaria entre os percentis 70 e 75 . Cole et al. ${ }^{20}$ sugeriram o uso de uma curva de IMC a partir de dados populacionais de várias regiões do mundo (Brasil, Estados Unidos, Holanda, Hong Kong, Reino Unido e Cingapura) e propuseram limites para sobrepeso e obesidade para a faixa etária de 2 a 20 anos para uso internacional.

O uso do IMC para identificação de sobrepeso/obesidade na adolescência é um instrumento adequado para inquéritos populacionais, entretanto, não mostra correlação entre composição corporal e maturação sexual. ${ }^{21} \mathrm{O}$ uso das dobras cutâneas poderia aumentar a especificidade na identificação dos adolescentes com obesidade, 22 contudo requer um examinador experiente para essa determinação, ficando difícil de ser realizado em trabalhos de pesquisa de campo.

Estudos de prevalência de sobrepeso/obesidade, utilizando amostras populacionais de adolescentes, foram realizados em outras regiões do país em épocas diferentes. Terres et al. ${ }^{23}$ realizaram um estudo transversal de base populacional com 960 adolescentes no município de Pelotas, Rio Grande do Sul, mostrando uma prevalência de sobrepeso/obesidade de 25,9\%. Vasconcelos e Silva, 24 analisaram 316.925 adolescentes masculinos com 18 anos nos Estados do Nordeste do Brasil e encontraram uma prevalência de sobrepeso/obesidade de $10,6 \%$. Castro et al. 25 estudando uma amostra de 1945 alunos adolescentes da rede municipal de educação do Rio de Janeiro, detectaram uma prevalência de sobrepeso/obesidade de $16,8 \%$. Salles et al.26 fizeram uma avaliação em 621 adolescentes das redes pública e privada, em Florianópolis, que mostrou uma prevalência de sobrepeso/obesidade nas escolas públicas de $13,1 \%$ e nas particulares de $7,6 \%$.

A prevalência de obesidade aqui encontrada pode ser considerada elevada se comparada com a maioria dos estudos nacionais citados, não chegando, entretanto, aos níveis de alguns países desenvolvidos, como os Estados Unidos, onde, na faixa etária de 12-19 anos, 15,5\% são obesos. 27

Alguns estudos demonstraram que fatores de risco para o aumento do peso na infância estão muito ligados à baixa atividade física e às características do meio familiar. 28 Deve ser ressaltado, também, o fato de que, em muitos países, ocorre o aumento no consumo de gordura e na densidade energética das alimentações. Nos países em desenvolvimento, o fenômeno da urbanização determina os padrões de atividade física e características dos alimentos. ${ }^{29}$

A prevalência do sobrepeso e da obesidade na população jovem começa a chamar atenção no Brasil. Evidências demonstram que a condição nutricional da criança brasileira apresenta modificações substanciais nos últimos 15 anos, com redução da taxa de desnutrição e aumento da obesidade infantil. 30

Por ser considerado um problema de saúde pública, é necessário melhor dimensionar as taxas de sobrepeso/obesidade na criança e no adolescente no âmbito nacional, para que uma maior vigilância seja feita a fim de conter a progressão de um dos maiores males deste século. ${ }^{8}$ Investigações devem ser feitas para avaliar a evolução desse problema, com o intuito de estabelecer, como rotina nos colégios, uma avaliação periódica para triagem e identificação de crianças e adolescentes com excesso de peso.

Reconhecendo que pouco se fez na intervenção 
da obesidade no período da adolescência no Brasil, mudanças devem ser implementadas no país para se enfrentar esta nova realidade.

Conclui-se que é relevante o problema do sobrepeso/obesidade em adolescentes escolares de Fortaleza, sendo maior nas escolas da rede privada e na adolescência precoce (10 a 14 anos), não apresentando diferença entre os sexos. A divulgação desses

\section{Referências}

1.WHO (World Health Organization). Obesity - preventing and managing the global epidemic. Report of a WHO consultation on obesity. Geneva; 1998.

2. Kosti RI, Panagiotakos DB. The epidemic of obesity in children and adolescents in the world. Cent Eur J Public Health. 2006; 14: 151-9.

3. Rubenstein AH. Obesity: a modern epidemic. Trans Am Clin Climatol Assoc. 2005; 116: 103-13.

4. De Onis M, Blossner M. Prevalence and trends of overweight among preschool children in developing countries. Am J Clin Nutr. 2000; 72: 1032-9.

5. Heald EP. Adolescent nutrition. Med Clin North Am. 1975; 59: 1329-36.

6. Guo SS, Chumlea WC. Tracking of body mass index in children in relation to overweight in adulthood. Am J Clin Nutr. 1999; 70: 145-8

7. Popkin BM. The Nutrition transition and obesity in the developing world. J Nutr. 2001; 131: 871-3.

8. Monteiro CA, Conde WL. Tendência secular da obesidade segundo estratos sociais: Nordeste e Sudeste do Brasil 1975-1989-1977. Arq Bras Endocrinol Metabol. 1999; 43: 186-94.

9. Neutzling MB. Sobrepeso em adolescentes brasileiros Brasil, PNSN-1989 [dissertação mestrado]. São Paulo: Escola Paulista de Medicina da Universidade Federal de São Paulo; 1998.

10. Magalhães VC, Azevedo G, Mendonça S. Prevalência e fatores associados a sobrepeso e obesidade em adolescentes de 15 a 19 anos das regiões Nordeste e Sudeste do Brasil, 1996 - 1997. Cad Saúde Pública. 2003; 19: 129-39.

11. Pereira RMM. Prevalência de obesidade em crianças e adolescentes em escolas públicas de Fortaleza [dissertação mestrado]. Fortaleza. Centro de Ciências da Saúde. Universidade Estadual do Ceará; 2002.

12. Instituto Nacional de Estudos e Pesquisas Educacionais. Censo escolar. Disponível em: htpp://www.inep.gov.br/ básica/censo/Escolar/Sinopse/ sinopse 2002. [2003 fev 15]. dados torna-se necessária para que se estabeleça um alerta à dimensão desse problema.

\section{Agradecimentos}

À Fundação Cearense de Apoio ao Desenvolvimento Científico e Tecnológico, pelo financiamento.
13. Must A, Dallal GE, Dietz WH. Reference data for obesity: $85^{\text {th }}$ and $95^{\text {th }}$ percentiles of body mass index (wt/ht 2$)$ and triceps skinfold thickness. Am J Clin Nutr. 1991; 53: 83946.

14. WHO (World Health Organization). Physical status: the use and interpretation of anthropometry. Report of a WHO Expert Committe. Geneva; 1995. p. 368-9. (Technical Report Series n. 854).

15. OMS (Organización Mundial de la Salud). La salud de los jóvenes: un reto y una esperanza. Geneva; 1995.

16. Sinaiko A. Obesidade, resistência à insulina e síndrome metabólica. J Pediatr (Rio J). 2007; 83: 3-4.

17. Luiz AMAG, Gorayeb R, Liberatore Junior RDO, Domingos NAM. Depressão, ansiedade e competência social em crianças obesas. Est Psicol (Natal). 2005; 10: 35-9.

18. Schofeld-Warden N, Warden $\mathrm{CH}$. Obesidade pediátrica: uma visão global da etiologia e do tratamento. Clin Pediatr Am Norte. 1977; 2: 346-66.

19. Sardinha LB, Going SB, Teixeira PJ, Lohman TG. Receiver operating characteristic analysis skinfold thickness and arm girth for obesity screening in children and adolescents. Am J Clin Nutr. 1999; 70: 1090-5.

20. Cole TJ, Bellizzi MC, Flegal KM, Dietz WH. Establishing a standard definition for child overweight and obesity worldwide: international survey. BMJ. 2000; 320: 1240-3.

21. Anjos LA. Epidemiologia da obesidade na infância. In: Fisberg M. Atualização em obesidade na infância e adolescência. São Paulo: Atheneu; 2004. p.11-7.

22. Cintra IP, Costa RF, Fisberg M. Composição corporal na infância e adolescência. In: Fisberg M. Atualização em obesidade na infância e adolescência. São Paulo: Atheneu; 2004. p. 27-40.

23. Terres NG, Pinheiro RT, Horta BL, Pinheiro KAT, Horta LL. Prevalência e fatores associados ao sobrepeso e à obesidade em adolescentes. Rev Saúde Pública. 2006; 40: 627-33. 
24. Vasconcelos VL, Silva GAP. Prevalências de sobrepeso e obesidade em adolescentes masculinos, no Nordeste do Brasil, 1980-2000. Cad Saúde Pública. 2003; 19: 445-51.

25. Castro IRR, Engstrom EM, Anjos LA, Azevedo AM, Silva CS. Perfil nutricional dos alunos da rede municipal de educação da cidade do Rio de Janeiro. In: Anais do Simpósio Obesidade e Anemia Carencial na Adolescência; 2000 jun 8-9, Salvador, Bahia. São Paulo: Instituto Danone; 2000. p. 232. [resumo]

26. Salles RK, Kazapi IAM, Di Pietro PF. Ocorrência de obesidade em adolescentes da rede de ensino do município de Florianópolis. In: Anais do Simpósio Obesidade e Anemia Carencial na Adolescência; 2000 Jun 8-9, Salvador, Bahia. São Paulo: Instituto Danone; 2000. p. 235. [resumo]
27. Ogden CL, Flegal KM, Carroll MD, Johnson CL. Prevalence and trends in overweight among US children and adolescents, 1999-2000. JAMA. 2002; 288: 1728-32.

28. Steinbeck KS. The importance of physical activity in the prevention of overweight and obesity in childhood: a review and an opinion. Obes Rev. 2001; 2: 117-30.

29. Popkin BM. The nutrition transition and its health implications in lower income countries. Public Health Nutr. 1998; 1: 5-21.

30. Silva RG. Condição nutricional de pré-escolares em escolas públicas do muncípio de São Carlos, SP de acordo com a condição socioeconômica [dissertação mestrado]. São Paulo: Departamento de Nutrição da Universidade Federal de São Paulo; 2001.

Recebido em 25 de agosto de 2006

Versão final apresentada em 3 de dezembro de 2006

Aprovado em 4 de fevereiro de 2007. 